\title{
Molecular dynamics modelling of nanocarbon cluster properties under conditions close to HE detonation
}

\author{
I.V. Derbenev, N.E. Chizhkova, F.A. Sapozhnikov, and V.V. Dremov ${ }^{a}$ \\ Russian Federal Nuclear Centre - Zababakhin Institute of Applied Physics (RFNC-VNIITF), \\ 456770 Snezhinsk, Russia
}

\begin{abstract}
We use molecular dynamics for modelling properties of carbon nanoclusters. The size of modelled carbon nanoclusters is below $5 \mathrm{~nm}$, which is typical of detonation diamond nanoclusters. We have found their structural changes at $\mathrm{P}=0$ to be as follows: Diamond $\rightarrow$ Diamond core + GL-surface $\rightarrow$ sandwich-type graphite $\rightarrow$ Graphite-like liquid. In smaller clusters the transformations start at a lower temperature. Adaptive Template Analysis (ATA) was used to determine the structures. We studied evaporation properties at temperatures above $5000 \mathrm{~K}$. For clusters of several thousands of atoms, the simple dependence $k_{\text {vap }} \sim e^{-T_{0} / T} / N^{1 / 3}\left(T_{0}\right.$ is constant) is quite good. It has been found out that densities of saturated vapour for clusters containing from 4000 to 8000 atoms are very close at $\mathrm{T}=$ $5000 \mathrm{~K}$.

The structure of nanoclusters was studied at nonzero pressures set by an argon environment. Calculated results suggest that the patterns for different temperatures are qualitatively similar for three pressures under study $(20,25$ and $30 \mathrm{GPa})$. At $\mathrm{T}=1000-1500 \mathrm{~K}$, the initial diamond core is preserved and a thin disordered GL layer is present on the surface. At $\mathrm{T}=2000-5000 \mathrm{~K}$, graphite grains form in the sample and a thin layer of liquid is present on its surface. The sample is amorphous at $5500 \mathrm{~K}$ and $6000 \mathrm{~K}$. The prevalence of the graphite phase at these pressures seems to come from the absence of long-range interaction in REBO-2002.
\end{abstract}

\section{Introduction}

The overwhelming majority of high explosives (HE) in practical use have much carbon and not enough oxygen for its oxidation which causes its condensation in solid phases. This phenomenon has often been observed in experiment [1-11] and is a subject of theoretical research including phenomenological studies with EOS (e.g., the BKW) and macrokinetics models [12] and more detailed studies into the physics of carbon condensation in detonation products, its phase states, and kinetics of carbon particle growth/vaporization (see, for example, [13]).

\section{Simulation of nanocluster structures at $\mathbf{P}=\mathbf{0}$}

To better understand how the clusters form, grow and influence detonation requires a more detailed study into their properties under detonation conditions. The method of molecular dynamics (MD) is well suited for this purpose and we used it here. The many-body REBO potential of the year 2002 [14] was used to model interaction between carbon atoms. We integrated Newton's equations with the Verlet algorithm. A time step was $0.2 \mathrm{fs}$.

\footnotetext{
a e-mail: i.v.derbenev@vniitf.ru
}

This is an Open Access article distributed under the terms of the Creative Commons Attribution-Noncommercial License 3.0, which permits unrestricted use, distribution, and reproduction in any noncommercial medium, provided the original work is properly cited. 


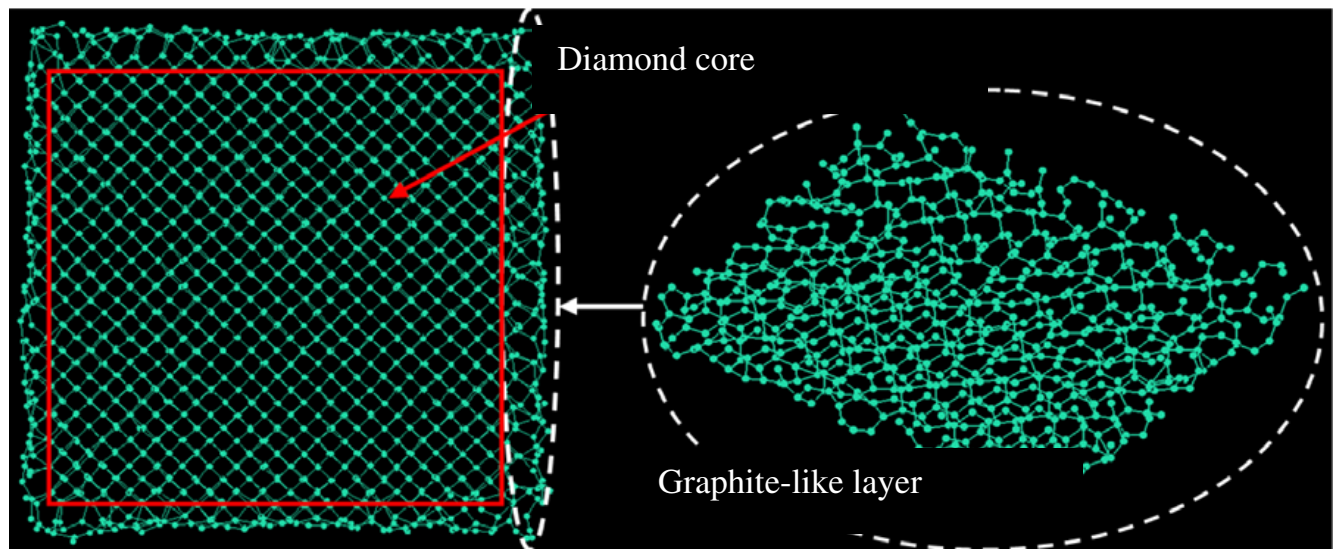

Fig. 1. A sample section at $\mathrm{T}=1000 \mathrm{~K}$.

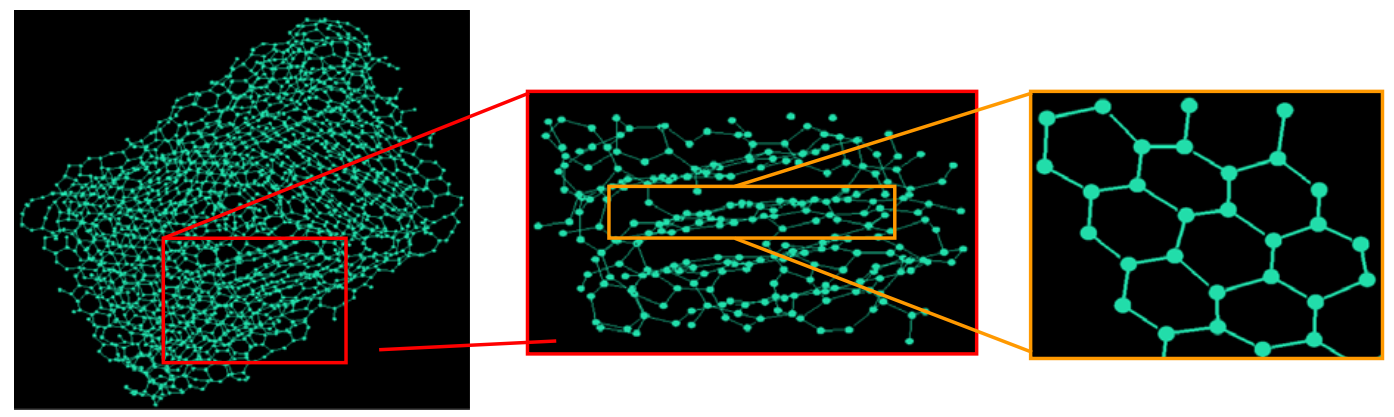

Fig. 2. A section of the 8000 -atom cluster, $T=3000 \mathrm{~K}$ : graphite polycristalline, layer graphite, graphene.

Properties of nanoclusters (initially with a diamond structure) were studied with respect to their size, pressure and temperature. The size was varied from 216 to 8000 atoms. Why this size? Because most nanodiamonds found in experiment are $3-5 \mathrm{~nm}$ in size (see, for example, [15]). The study of smaller clusters is of importance for understanding the processes of their growth. Temperatures of interest are between $1000 \mathrm{~K}$ and $6000 \mathrm{~K}$.

The study of cluster structures rises a problem of phase state detection. The problem was resolved with the method of Adaptive Template Analysis (ATA) [16] which proved to perform very well. The method was developed at RFNC-VNIITF. We also used the analysis of pair correlation functions and the visual analysis of the structures generated.

We modeled clusters at temperatures between 1000 and $6000 \mathrm{~K}$ during 20 ps.

At temperatures from 1000 to $1500 \mathrm{~K}$, the clusters preserve their diamond core and a graphite-like (GL) phase is produced on the surface. This is shown in Fig 1.

We can see the surface of GL-layer immediately: atoms have mainly three neighbours ( $\mathrm{sp}^{2}$ - hybridization) which is typical of graphite. Analysis of the correlation function $g_{2}(r)$ of surface atoms shows that their structure is closer to that of graphite. At $1500 \mathrm{~K}$, the GL-layer is thicker and the diamond core is smaller.

As temperature increases first to $2000 \mathrm{~K}$ and then to $4000 \mathrm{~K}$, the diamond core transforms to sandwich-type graphite with differently oriented domains (see Fig. 2).

At temperatures from 4000 to $4500 \mathrm{~K}$, sandwich-type graphite transforms, partly or completely, into the amorphous phase.

Our modelling of isolated nanoclusters in vacuum has eventually shown that with the increasing temperature, the structures transform in a quite predictable way, specifically:

Diamond $\rightarrow$ Diamond core + GL-surface $\rightarrow$ sandwich-type graphite $\rightarrow$ GL-liquid

For smaller clusters, the transformation starts at a lower temperature. 


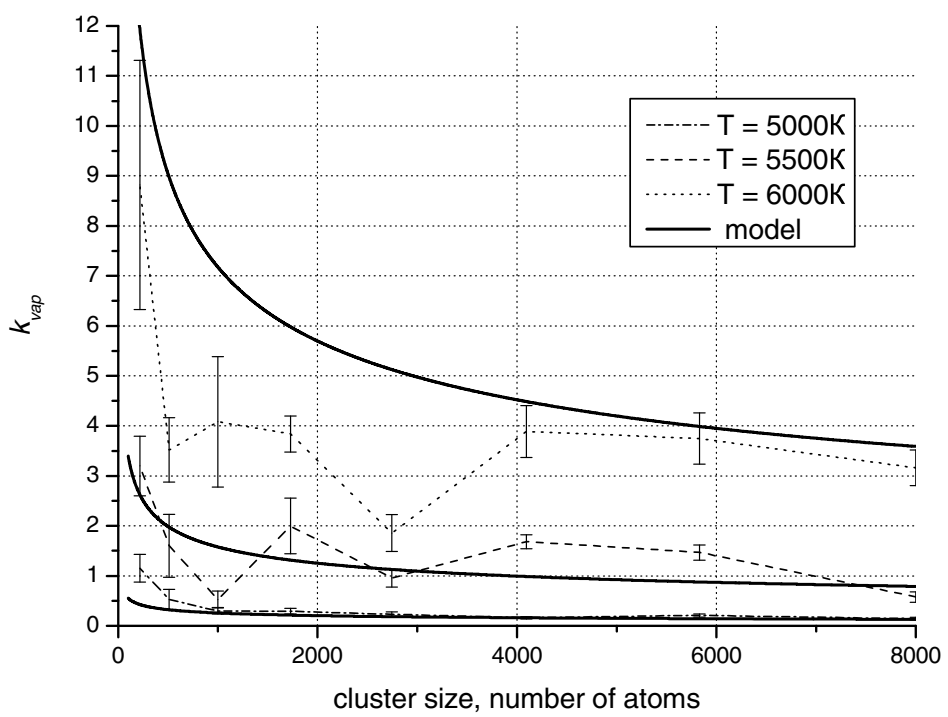

Fig. 3. Evaporation rate $k_{\text {vap }}(d)$ for three temperatures: $5000 \mathrm{~K}, 5500 \mathrm{~K}$, and $6000 \mathrm{~K}$; and a model $k_{\text {vap }} \sim$ $e^{-T_{0} / T} / N^{1 / 3}$.

\section{Simulation of evaporation properties}

At temperatures $5000 \mathrm{~K}$ and higher, the evaporation of atoms from the surface starts to play a great role. The higher the temperature, the more intense the evaporation is, and the smaller the cluster, the more atoms are in the surface layer from which they are evaporated and the higher the rate of their evaporation.

Evaporation rates versus cluster size and temperature were found.

Here the model $k_{\text {evap }}$ is a function of form $k_{\text {vap }} \sim e^{-T_{0} / T} / N^{1 / 3}\left(T_{0}=10000 \mathrm{~K}\right)$, which is dependent on temperature in the Arrhenius form, as in [17], which is good enough for quite big clusters, and on the number of atoms as $S / V$. For small clusters that are studied in this work, this model is too rough which increases the value of their direct modelling with MD methods. The peak of the size distribution of evaporated molecules $C_{n}$ is above $n=3-4$.

\section{Direct simulation of saturated vapour}

The next step is the direct modelling of vapour-cluster balance. We put a cluster in GL-phase into the centre of a box of certain size and put vapour of some density around the cluster. The size distribution of vaporised molecules corresponds to that one obtained in the above calculations. Our calculations of vapour-cluster balance at $T=5000 \mathrm{~K}$ were done for clusters of different sizes. The vapour-cluster balance was achieved by iterative density fitting rather than by "direct" evaporation that would be very expensive. The size distribution of saturated vapour differs from that of evaporated molecules and its maximum falls on 12-atomic molecules. The densities of saturated vapour $\left(\rho_{s v}\right)$ for clusters of sizes from 4000 to 8000 were found to be almost identical (see Table 1).

It should also be noted that evaporation rates for clusters of 8000,5832 and 4096 atoms $T=5000 \mathrm{~K}$ are equal within calculation accuracy:

$$
\begin{aligned}
& k_{\text {vap }}(8000,5000 \mathrm{~K})=0,1409 \pm 0,0277[\operatorname{atom} / \mathrm{ps} /(d=8000)]^{*} 100 \% \\
& k_{\text {vap }}(5832,5000 \mathrm{~K})=0,2065 \pm 0,0281[\operatorname{atom} / \mathrm{ps} /(d=5832)]^{*} 100 \% \\
& k_{\text {vap }}(4096,5000 \mathrm{~K})=0,1590 \pm 0,0262[\operatorname{atom} / \mathrm{ps} /(d=4096)]^{*} 100 \%
\end{aligned}
$$


EPJ Web of Conferences

Table 1. Density of saturated vapour at $T=5000 \mathrm{~K}$.

\begin{tabular}{|c|c|}
\hline Size of cluster, atoms & Saturated vapour density $\rho_{s v}, \mathrm{~g} / \mathrm{cm}^{3}$ \\
\hline 4096 & 0.0263 \\
\hline 5832 & $0.0236-0.0262$ \\
\hline 8000 & 0.0230 \\
\hline
\end{tabular}

The linear dimensions of clusters, which characterize the "absorption cross-section" of vapour, differ by no more than $25 \%$.

Small differences in evaporation rates and linear dimensions explain why the values of $\rho_{s v}$ are close for clusters of 8000, 5832, and 4096 atoms at $T=5000 \mathrm{~K}$. At lower temperatures, differences in $\rho_{s v}$ 's will be yet smaller because differences in $k_{v a p}$ will be smaller too (it is seen from Figure 3), but differences in geometric sizes will remain approximately the same. We can say with confidence that the growth/evaporation induced change in the size of a 4000-8000-atom carbon cluster is not a problem parameter but dominated by temperature. Since only partial pressure is important for growth/vaporization and $\rho_{s v}$ is known, we can see what happens under specific thermodynamics conditions - condensation and growth of carbon drops or their evaporation.

Undoubtedly, carbon $\rho_{s v}$ at specific temperatures is an important but not a unique parameter which defines the fate of clusters with the size of interest. Also of great importance is the chemical influence of the gaseous environment (detonation products). There is, for example, a hypothesis that cluster's properties (structure, evaporation rate etc.) might significantly change if its surface is partly covered with other atoms or radical groups.

\section{Simulation of nanocluster structures at $\mathrm{P} \neq 0$}

The cluster's phase state is crucial for growth/vaporization. If a cluster is liquid (always present in the surface layer at $P=0$ ), it will absorb vapour with a much greater probability than a cluster with the solid surface. So there arises a reasonable question about the phase diagram of carbon nanoclusters in the range of thermodynamic conditions of interest. That is why an attempt was made to study the structure of nanoclusters at a nonzero external pressure which was set by adding an argon environment.

Ar-Ar and Ar-C interactions were modelled with a simple Lennard-Jones (LJ) potential which describes argon properties well and has the following parameters: $\alpha=13.57, r^{*}=3.791 \cdot 10^{-10} \AA$, $\varepsilon=1.68 \cdot 10^{-21} \mathrm{~J}$. The pressure and temperature ranges are, respectively, $20-30 \mathrm{GPa}$ and $1000-6000 \mathrm{~K}$. Our simulations show that a thin layer of liquid forms on the nanocluster's surface.

Calculated results suggest that the patterns for different temperatures are qualitatively similar for three pressures under study $(20,25$ and $30 \mathrm{GPa})$.

At $\mathrm{T}=1000-1500 \mathrm{~K}$, the initial diamond core is preserved and a thin disordered GL layer is present on the surface. At $\mathrm{T}=2000-5000 \mathrm{~K}$, graphite grains form in the sample and a thin layer of liquid is present on its surface. The sample is amorphous at $5500 \mathrm{~K}$ and $6000 \mathrm{~K}$. The prevalence of the graphite phase at these pressures seems to come from the absence of long-range interaction in REBO-2002.

\section{References}

1. A.I. Lyamkin, E.A. Ershov and A.P. Petrov. Getting diamonds from HE. Transactions of Academy of Sciences (in Russian), V. 302, No.3, P. 611-613. (1988).

2. Huang Fenglei, Tong Yi, Yun Shourong. Synthesis mechanism and technology of ultrafine diamond from detonation. J. Solid State Physics (in Russian), V.46, Is. 4, P. 601-604. (2004).

3. A.E. Alexeyevski, M.V. Vul and V.I. Siklitski. Structure of diamond nanocluster. J. Solid State Physics (in Russian), V.41, Is. 4, P.740-743. (1999). 
4. V.M. Titov, V.F. Anisichkin and I.U. Malkov. Investigation of the synthesis process of superdispersed diamond in detonation wave. J. Combustion and Explosion Physics (in Russian), V.25, No.3, P.117-126. (1989).

5. V.N. Kolomiichuk, I.U. Malkov. Investigation of the synthesis of superdispersed diamond phases at detonation of mixture compounds. J. Combustion and Explosion Physics (in Russian), V.29, No.1, P.120-128. (1993).

6. A.V Kurdumov, O.N. Breusov, V.N. Drobishev, V.A. Melnikova and V.F. Tacii. About crystal structure of detonation diamonds. J. Combustion and Explosion Physics (in Russian), V.25, No.3, P.117-126. (1989).

7. K.V. Volkov, V.V. Danilenko and V.I. Elin. Synthesis of diamond from HE detonation products carbon. J. Combustion and Explosion Physics (in Russian), No., P.123-125. (1990).

8. A.L. Vereschagin, G.V. Sakovich, L.A. Petrova, V.V. Novoselov and P.M. Brilakov. Investigation of surface chemistry of superdispersed detonation diamond. Transactions of Academy of Sciences (in Russian), V.315, No.1, P.104-105. (1990).

9. B.A. Viskubenko, V.V. Danilenko, E.E. Lin, V.A. Mazanov, T.V. Serova and A.P. Tolochko. Influence scale factor on sizes and quantity detonation syntheses diamond. J. Combustion and Explosion Physics (in Russian), V.28, No.2, P.108-109. (1992).

10. V.V. Danilenko. Phase diagram of nanocarbon. J. Combustion and Explosion Physics (in Russian), V.41, No.4, P.110-116. (2005).

11. V.V. Danilenko. Peculiarity of synthesis of detonation diamond. J. Combustion and Explosion Physics (in Russian), V.41, No.5, P.104-116. (2005).

12. Konstantin F. Grebenkin, Mikhail V. Taranik, Alexey L. Zherebtsov. Computer modelling of scale effects at heterogeneous HE detonation. 13th International DETONATION SYMPOSIUM (2006).

13. M.S. Shaw and J.D. Johnson. Carbon clustering in detonations, J. Appl. Phys. 62(5), p.2080 (1987).

14. D.W. Brenner, Olga A. Shendorova, Judith A. Harrison, Steven J. Stuart, Boris Ni and Susan B. Sinnott. J. Phys.: Condens. Matter 14 783-802 (2002).

15. V.D. Andreev and U.I. Sozin. Structure of superdispersed diamond. J. Solid State Physics (in Russian), V.41, Is. 10, P.1890-1893. (1999).

16. F.A. Sapozhnikov, G.V. Ionov and V.V. Dremov. Adaptive template analysis for identification of crystalline structures and defects in molecular-dynamics modelling of high rate straining. J. Chemical Physics (in Russian), V.27, No.3, P.63-70 (2008).

17. R.S. Berry and B.M. Smirnov. Phase transitions and associated phenomena in simple systems of bound atoms. J. Achievements of Physical Sciences (in Russian), V.175, No.4, P.367. (2005). 